\title{
The Use of Static Pressures of Heavy Gases Within a Quadrupole Ion Trap
}

\author{
Ryan M. Danell,* Allison S. Danell,* and Gary L. Glish \\ Department of Chemistry, University of North Carolina, Chapel Hill, North Carolina, USA
}

\author{
Richard W. Vachet \\ Department of Chemistry, University of Massachusetts, Amherst, Massachusetts, USA
}

\begin{abstract}
The performance of quadrupole ion traps using argon or air as the buffer gas was evaluated and compared to the standard helium only operation. In all cases a pure buffer gas, not mixtures of gases, was investigated. Experiments were performed on a Bruker Esquire ion trap, a Finnigan LCQ, and a Finnigan ITMS for comparison. The heavier gases were found to have some advantages, particularly in the areas of sensitivity and collision-induced dissociation efficiency; however, there is a significant resolution loss due to dissociation and/or scattering of ions. Additionally, the heavier gases were found to affect ion activation and deactivation during MS/MS, influencing the product ion intensities observed. Finally, the specific quadrupole ion trap design and the ion ejection parameters were found to be crucial in the quality of the spectra obtained in the presence of heavy gases. Operation with static pressures of heavy gases can be beneficial under certain design and operating conditions of the quadrupole ion trap. (J Am Soc Mass Spectrom 2003, 14, 1099-1109) ( 2003 American Society for Mass Spectrometry
\end{abstract}

$\mathrm{T}$ The quadrupole ion trap mass spectrometer (QITMS) comprises a large and continually growing segment of mass spectrometry. The QITMS possesses many advantages over other mass analyzers, including ease of use, size, cost, and ability to perform $\mathrm{MS}^{\mathrm{n}}$ analysis. With this greatly increased usage comes the motivation to enhance and extend its abilities. One area that has seen sporadic investigation is the buffer gas used within QITMS. The use and function of the buffer gas has been of interest to researchers for many years prior to commercialization of the QITMS [1-3].

The buffer gas is an important component of the QITMS and can affect many aspects of its operation. When injecting ions into the QITMS, one function of the buffer gas is to reduce ion kinetic energy. As the ions enter the trapping volume, they will encounter neutral buffer gas atoms or molecules and collide with them. One possible outcome of these collisions is the transfer of kinetic energy from the ions to the neutral target (the buffer gas). This reduction of ion kinetic energy is termed collisional cooling, and the process aids in ion trapping and therefore can improve sensitivity. Many collisions can occur once the ion is nominally trapped, yielding a condensed cloud of ions close to the center of

Published online August 11, 2003

Address reprint requests to Dr. G. Glish, Department of Chemistry, University of North Carolina, CB No. 3290, Venable Hall, Chapel Hill, NC 27514, USA. E-mail: glish@unc.edu

${ }^{*}$ Current address: The Rowland Institute at Harvard, 100 Edwin H. Land Blvd., Cambridge, MA 02142, USA. the trapping volume. With all of the ions condensed to the center of the QITMS and possessing similarly low kinetic energies, mass analysis can be performed with higher resolution and sensitivity [3].

A collision also can result in ion kinetic energy being converted into ion internal energy. The maximum amount of internal energy that can be deposited into the ion in a single collision is the center-of-mass kinetic energy, $E_{\text {com }}$, and can be calculated using the following eq:

$$
E_{c o m}=E_{l a b}\left(\frac{M_{n}}{M_{p}+M_{n}}\right)
$$

where $M_{n}$ is the mass of the neutral buffer gas atom or molecule, $M_{p}$ is the mass of the ion, $E_{l a b}$ is the ion's laboratory frame kinetic energy and $\mathrm{E}_{\mathrm{com}}$ is the centerof-mass kinetic energy. Increasing the internal energy of an ion is the desired outcome when performing collision-induced dissociation (CID). The most common way to implement CID in the QITMS is resonance excitation. Resonance excitation involves kinetically exciting specific ions of interest by applying a supplementary oscillating electric field at the frequency of the ions' motion. During the resonant excitation process, ions undergo collisions with the buffer gas. These collisions convert some of the ions' kinetic energy to internal energy. After enough kinetic energy has been transferred so that the internal energy exceeds the critical 
energy of dissociation, the parent ions can fragment. The resulting product ions are not in resonance with the supplementary electric field and subsequent collisions between the buffer gas and the product ions will, on average, reduce the product ion internal and kinetic energy. Collisionally cooling the product ions can enhance the MS/MS collection efficiency, but also will limit the variety of product ions formed. The dual functionality of the buffer gas in a QITMS enables the instrument to achieve MS/MS efficiencies that can approach $100 \%$ [4].

Ions need to be ejected from the QITMS to reach the detector, which is external to the trapping volume, and the buffer gas can also affect ion ejection. Ejection is most commonly accomplished with resonance ejection. Resonance ejection utilizes the same process as resonance excitation uses for CID except the ions are kinetically excited much faster, causing them to be ejected from the trapping volume before undergoing sufficient collisional activation. In some cases before the ion oscillations are large enough to eject the ion, it can undergo sufficient collisions with the buffer gas to cause the ion to dissociate. Scattering is another possible result from a collision. A dissociative collision will produce a smaller mass ion, which may be immediately ejected from the QITMS. A scattering collision can alter the phase relationship of the ion motion and the rf field, also causing the ion to be quickly ejected. Therefore, either outcome from a collision may modify the ejection time causing the ion to be ejected earlier than the majority of ions of the same $\mathrm{m} / \mathrm{z}$ [5]. As the mass spectrum is acquired over time, the difference in ejection time will degrade the resolution $(\mathrm{m} / \Delta \mathrm{m})$ of the mass spectrum.

Usually the buffer gas of choice in the QITMS is helium because it gives the best performance in terms of resolution and sensitivity. Helium has a large enough mass to produce significant collisional cooling; however, helium is not large enough to cause a significant amount of scattering or dissociation during ejection. The resulting mass spectra acquired with helium as the buffer gas generally have good resolution and peak shapes. However, there are reasons to use alternative, heavier buffer gases. The foremost of these is the added collisional cooling and collisional activation possible. As shown by eq 1 , if $M_{n}$ increases, $E_{\text {com }}$ also increases. Selection and proper use of a gas other than helium could conceivably enhance the operation of the QITMS.

Many experiments have been performed using pure gases other than $\mathrm{He}$ as well as mixtures of two different gases. In addition, both static and pulsed pressures have been used to further investigate the role of the buffer gas during different parts of the instrument's operation. The first experiments to investigate the performance of the QITMS with buffer gases other than He involved using low, static pressures of pure Ar [6]. These experiments were performed with standard geometry Finnigan ITMS electrodes and the research concentrated on the MS/MS performance of the
QITMS. In general, increased levels of internal energy deposition were observed with Ar over all pressures tested. The internal energy deposition exhibited a pressure dependence when He was used, with lower pressures yielding higher internal energies. The product ion intensities and mass resolution were lower with Ar, and this decrease in performance was postulated to be due to the increase in buffer gas mass serving to alter the ion ejection profile as discussed above. One earlier report noted that no ion signal was observed when Ar was used alone at higher pressures as a static buffer gas [7]. This latter experiment differed from previous heavy gas experiments in that the ions were injected into the QITMS rather than being formed in it.

Several researchers have also explored the option of mixed gases [7-11]. In most cases, the heavy gas was added to the standard operating pressure of He buffer gas. The buffer gas mixtures used were between 1-50\% heavy gas, and resulted in an overall greater total buffer gas pressure than usual. A problem with decreased sensitivity was observed in some cases [7, 11]; however, an increase in the sensitivity (termed the trapping efficiency in this earlier report) was observed for large CsI cluster ions [9]. Ion/neutral collisions still degraded the resolution even though the heavy gas pressure was reduced compared to the original experiments $[9,11]$. In all cases, an increase in the internal energy deposition during CID was observed once the optimal proportion (system dependent) of heavy gas was added to the standard helium pressure.

At this point, it is useful to make a note about terminology. When assessing the performance of the QITMS with heavy gases, it is often useful to compare the resulting signal intensity observed when the QITMS is operated with and without heavy gas added. This can give an indication of the effect the heavy gas has on the sensitivity of the instrument. In the past, however, several researchers have termed this measurement the trapping efficiency. A problem with the term trapping efficiency is that it can have several different meanings in the context of the QITMS. It can mean the efficiency of collecting ions that are injected into the QITMS, and it can be used to mean the efficiency with which those ions are stored in the QITMS (also termed the storage efficiency). Another problem with the term trapping efficiency is that in most cases the researchers are not actually measuring a physical value consistent with either of the above definitions. In most cases the observed signal is used as a measure of the trapping efficiency. However, several processes besides the trapping efficiency influence the observed signal intensity. These processes include, but are not limited to, ion injection efficiency, storage efficiency, and detection efficiency. There are few examples known where the necessary experimental steps were taken to measure the true trapping efficiency [12], and none of the previous 
heavy buffer gas research include these measurements. Therefore, throughout this paper the term sensitivity will be used when discussing comparisons of the ion signal produced with various experimental setups.

After the experiments with static pressures of heavy buffer gases indicated the loss in resolution, two research groups investigated the use of pulsed heavy gases $[8,13]$. In these experiments, the standard operating pressure of He was always present. The heavy gas was then pulsed into the instrument, adding to the $\mathrm{He}$ base pressure for a short time. The pulsing scheme was designed such that the heavy gas was only present for a specific segment of the experiment. When heavy gases were pulsed in during ion accumulation, a decrease in sensitivity was observed [13]. Although this agrees with the previous heavy buffer gas results discussed above $[7,11]$, a significantly different trapping scheme was used in the pulsed experiments. Additionally, in the former experiments the heavy gas was present for the entire experiment, but it was only present for ion accumulation in the pulsed gas experiment. Therefore, the decreases in sensitivity observed are most likely due to different effects. When heavy gases were pulsed in during the analytical ramp segment of the experiment, the resolution was degraded. The drop in resolution was again attributed to ion/neutral collisions. More importantly, however, was the finding that when the heavy gases were pulsed in during CID, the MS/MS efficiency increased and additional product ion types were detected. This result was attributed to an increase in the internal energy deposition and was observed without any degradation of the performance (i.e., sensitivity and resolution) of the QITMS.

Previous work with heavy gases indicates that they can improve performance under certain operating conditions. It also has been shown that heavy gases affect different segments of the experiment in different ways. However, all of the earlier research was performed with first generation commercial QITMS instrumentation. This generation of QITMS did not utilize higher order fields to the extent that current generation instruments do. For these reasons, the performance of three commercial QITMS has been investigated using a range of static pressures of heavy gases. Static pressures of individual heavy gases (no He added) were used to simplify the experiment so that the fundamental effects of the heavy gas alone could be analyzed. Argon was the primary heavy gas investigated, but ambient air also was used due to its potential application in fieldbased miniature mass spectrometers. Additionally, the use of no added buffer gas (just the air introduced from the external ionization source) also was investigated. The standard metrics for MS and MS/MS performance were evaluated, along with parameters specific to QITMS instruments, such as $\beta_{\text {eject }}$ and higher order fields. In all cases the performance was compared to that with pure $\mathrm{He}$ as the buffer gas.

\section{Experimental}

\section{Instruments}

Three different QITMS instruments were used in the experiments described here. A Bruker (Billerica, MA) Esquire ion trap mass spectrometer was used for most experiments. The use of this instrument should be assumed unless otherwise noted. The geometry and spacing of the electrodes in the Esquire ion trap have been designed to enhance the contribution from higher order (specifically hexapolar) fields [14, 15]. This instrument is equipped with a standard Analytica (Branford, CT) electrospray ionization source that has been slightly modified to utilize a more durable custom glass transfer capillary.

The second instrument was a highly modified Finnigan (San Jose, CA) ITMS that was used in a previous heavy gas study [8]. This instrument is equipped with a custom nanoESI source. The third QITMS used was a commercial Finnigan LCQ, with the standard Finnigan ESI source interface. This instrument has been modified so that a supplemental ac voltage can be applied to the endcap electrodes with a waveform generator [16]. In the context of the current research, the use of a waveform generator provides the ability to select the $\beta_{\text {eject }}$ value used during the analytical scan.

\section{Gases}

Research grade gases were used in all experiments, except when ambient laboratory air was used. All gases were leaked into the vacuum systems through precision needle valves and pressures were set and stabilized before any experiments were initiated. Pressures were measured with either a Penning or Bayard-Alpert type ionization gauge, depending on the instrument. Buffer gas pressures were set as the static pressure added to the ultimate base pressure, all measured with the inlet on the electrospray source closed. The base pressures of the vacuum systems used here were typically in the $10^{-7}-10^{-6}$ torr range. Gases were always introduced directly into the trapping volume; however, pressures were not measured within the trapping volume, as it is experimentally difficult to perform such a measurement. In all cases, the vacuum gauge was located some distance (usually between 15 and $20 \mathrm{~cm}$ ) away from the analyzer. The Finnigan ITMS trapping volume is open as only ceramic rod spacers are used to separate the electrodes. The LCQ ion trap was modified to be similarly open, meaning that besides the electrodes, there is nothing else that blocks gas flow in and out of the trapping volume. Therefore, the pressures measured by the vacuum gauges on the ITMS and the LCQ instruments were representative of the actual pressures within the trapping volumes. However, this was not the case for the Esquire ion trap. The Esquire uses solid electrode spacers to tightly enclose the trapping volume making the gas flow in and out conductance limited. As 
a result, when a buffer gas was being introduced into the analyzer, the vacuum gauge indicated a pressure lower than the actual pressure the trapped ions were experiencing.

The difference between the actual and measured pressures in the Esquire motivated further investigation toward developing a calibration curve for converting between the two pressure values. The calibration was attempted using a gas phase proton-transfer reaction between cytochrome $c$ and dimethylamine [17]. The rate of the ion/molecule reaction at various measured pressures of dimethylamine [18] was used to calculate the analyzer pressure. A complete set of the experiments needed to develop a calibration curve covering the desired range was not possible due to rate of the ion/molecule reaction in this pressure range. However, the successful experiments indicated that the actual pressure in the analyzer is approximately 100 times greater than that measured by the gauge in the main vacuum chamber. The rough calibration accounts for the differences in the operating pressure of Esquire ion trap and that of other QITMS. The Esquire normally operates with $2.1 \times 10^{-5}$ torr of $\mathrm{He}$, as measured by the vacuum gauge. From the above calibration, the actual pressure within the trapping volume is approximately 2 $\times 10^{-3}$ torr, whereas other QITMS usually operate with $1 \times 10^{-3}$ torr of He. Because a calibration curve could not be established, the pressures reported for the Esquire ion trap throughout this work are those measured by the gauge. Based on the above experiments these reported pressures are approximately two orders of magnitude lower than the actual pressure in the analyzer trapping volume.

The buffer gas pressure ranges considered with the Esquire ion trap are as follows (measured outside of the trapping volume): He and $\mathrm{Ar}=0.71-7.1 \times 10^{-5}$ torr, air $=0.71-6.4 \times 10^{-5}$ torr. The standard operating pressure for this instrument with $\mathrm{He}$ is $2.1 \times 10^{-5}$ torr. All reported pressures throughout this paper are added pressure to the ultimate base pressure of the instrument when the source is closed, and all pressures are corrected for the specific response of the vacuum gauge to the measured gas. Some experiments on the Esquire ion trap were performed without any buffer gas added to the trapping volume. This state will be represented by a point at zero pressure in the plots discussed below (marked with a solid triangle). However, this state is not actually "zero pressure". Without any added buffer gas, the only gas present in the trapping volume is the laboratory air that enters through the source and is not pumped away by the rough and first high vacuum regions; therefore, the "zero pressure" point can be interpreted as a very low added pressure of air. The ultimate base pressure reached in this instrument with the source closed was approximately $1.5 \times 10^{-6}$ torr. The added pressure from laboratory air with the source open was measured to be approximately $3.7 \times 10^{-5}$ torr. The air was present in all experiments conducted on the Esquire. The pressure from air inside the trap- ping volume is assumed to be similar to that measured by the vacuum gauge due to diffusion within the vacuum system and the factor of 100 noted above does not apply.

\section{Solutions/Samples}

The peptide Leucine Enkephalin (sequence YGGFL, abbreviated Leu-Enk), obtained from Sigma Chemical Company (St. Louis, MO), was used for all experiments. Solutions resulting in final concentrations between 20 $250 \mu \mathrm{M}$ were prepared in a 75:20:5 mixture of methanol: water:acetic acid (glacial).

\section{Instrument Operating Conditions}

After first obtaining electrospray signal, the various source voltages were adjusted to minimize fragmentation and maximize the overall intensity of the $[\mathrm{M}+\mathrm{H}]^{+}$ species. For MS/MS experiments, the amplitude of the resonance excitation voltage was adjusted such that the product ion signal was maximized, yielding the maximum MS/MS efficiency [19]. MS and MS/MS data were obtained from averages of approximately 25 scans.

\section{Results}

\section{MS Performance}

Mass spectra of Leu-Enk obtained with $\mathrm{He}$ and Ar as the buffer gas are shown in Figure 1. Qualitatively the spectra look very similar, and little additional fragmentation or anomalous peaks are observed when using Ar as the buffer gas. The inset included in Figure 1 shows that the resolution has degraded with the use of Ar as the isotopic peaks are no longer resolved. The decrease in resolution observed is in agreement with previous results obtained with static pressures of heavy gases in QITMS [6]. Additionally, the inset shows that the apparent mass of the protonated molecule has shifted when Ar is the buffer gas. This is because the instrument requires a separate calibration for Ar (the active calibration is based on using He as the buffer gas) and indicates that the presence of the heavier bath gas causes ions to be ejected sooner.

The resolution loss was quantified at different buffer gas pressures, and the results are shown in Figure 2. It should be noted that the resolution cannot be determined just by measuring the peak width because the ${ }^{13} \mathrm{C}$ isotope peak is no longer resolved from the ${ }^{12} \mathrm{C}$ isotope peak with Ar as the buffer gas (see Figure 1). The reduced resolution observed with Ar is expected to increase the error in the FWHM measurement used to create Figure 2 because the peak shape is not perfectly gaussian. The larger FWHM values have errors of $10-15 \%$ associated with them. Gaussian peak shape modeling was used to deconvolute the contribution from the isotope peak to the peak widths observed. The 


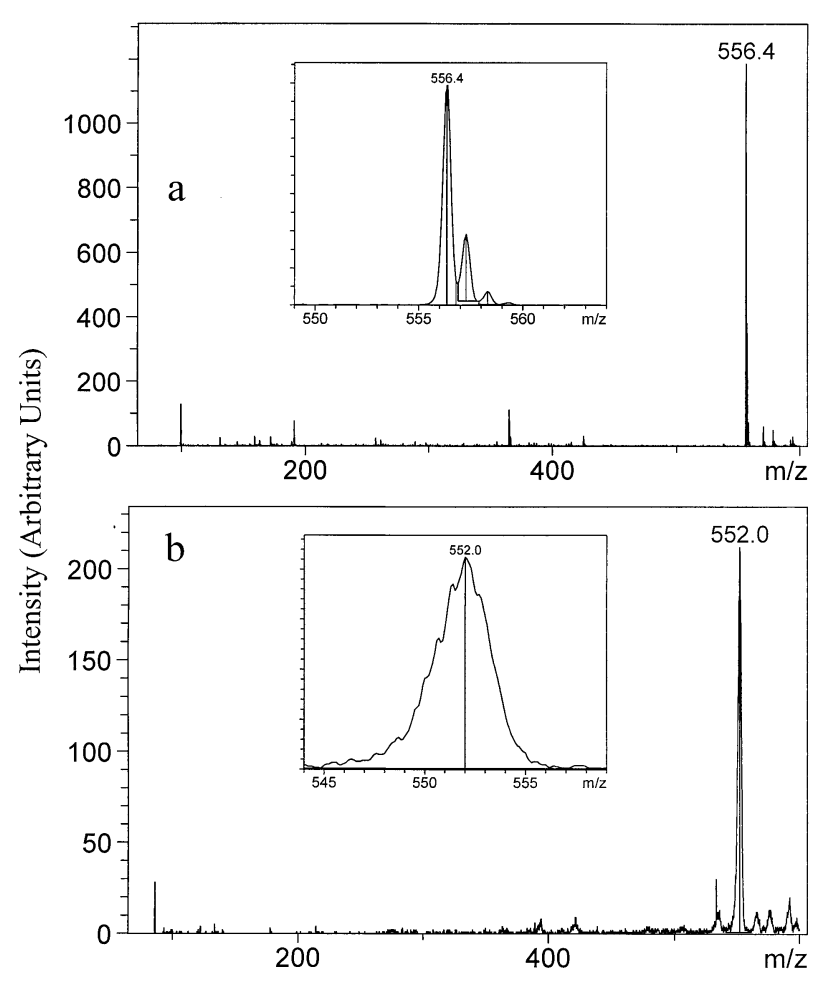

Figure 1. Mass spectra of Leu-Enk in $2.1 \times 10^{-5}$ torr of (a) He and (b) Ar. The insets show the protonated molecule region of the mass spectrum. Note: The instrument was not recalibrated for data taken with $\mathrm{Ar}$ as the buffer gas.

resolving power (defined as $\mathrm{m} / \Delta \mathrm{m}$ ) of the instrument was found to drop from 1400 (at $m / z$ 556) with He to 250 or less with Ar. Therefore, the resolving power drops by at least a factor of 5.6 when Ar is used. The resolution was degraded further as the pressure was increased over the range investigated. The resolution degradation was not as severe with air as the buffer gas, but the peak widths were still wider than those observed with He. Finally, it was observed that with no added buffer gas

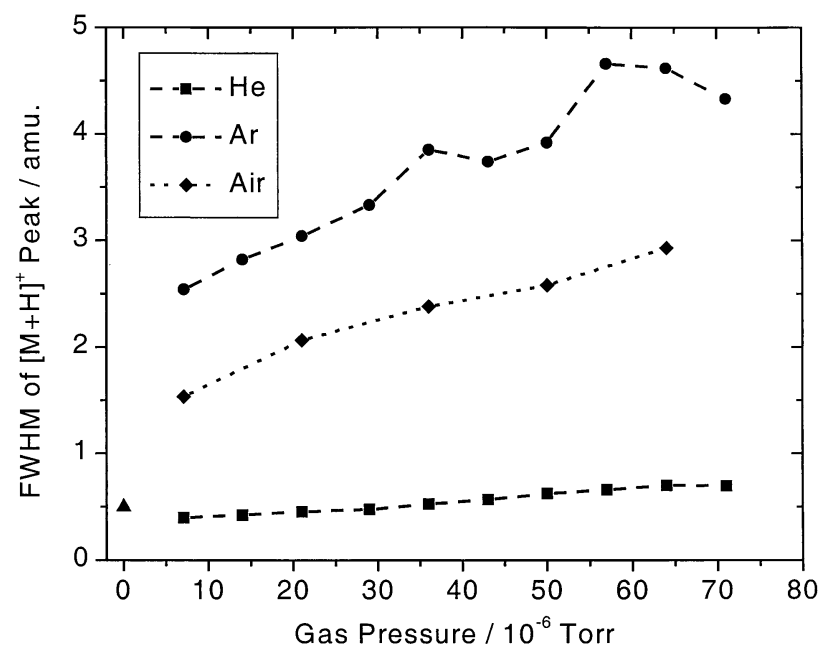

Figure 2. Resolution loss of the Leu-Enk protonated species, as a function of pressure for $\mathrm{He}, \mathrm{Ar}$, and Air.

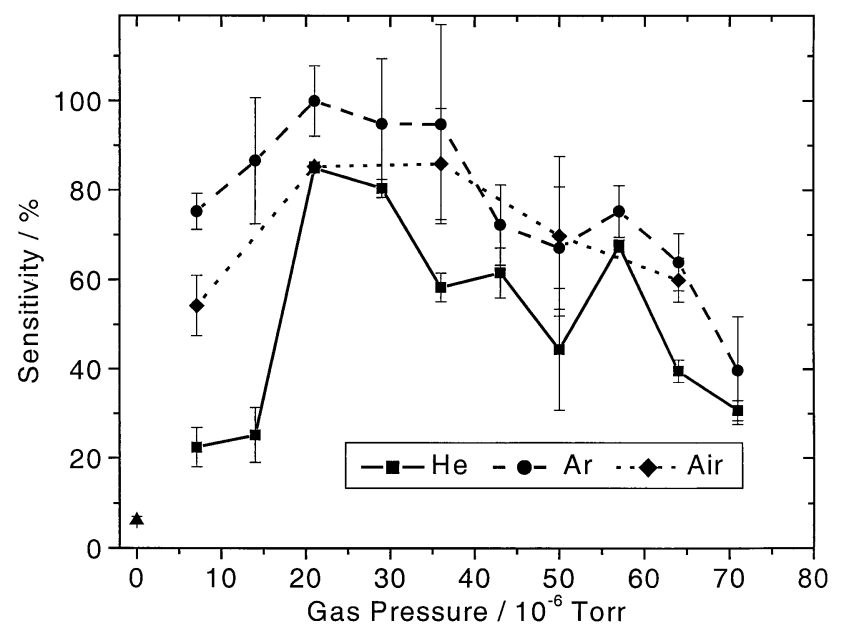

Figure 3. Sensitivity, defined as the signal intensity obtained from Leu-Enk protonated molecules in a given time interval and normalized to the maximum value obtained, as a function of pressure for He, Ar, and Air.

present the resolution was quite good, although not as good as with low pressures of He. It must be remembered that when no buffer gas is added, there is still a base pressure of air present in the vacuum system as well as in the trapping volume. Once a buffer gas is introduced, however, the gas in the trapping volume will effectively be the pure added buffer gas and not the air introduced from the electrospray source. The observed changes in resolution support the belief that heavier buffer gases degrade resolution by dissociating or scattering analyte ions during mass analysis. These effects are minimized with $\mathrm{He}$ as the buffer gases because of its small mass.

The sensitivity for $[\mathrm{M}+\mathrm{H}]^{+}$ions from Leu-Enk was also evaluated in the presence of heavy gases. The results are shown in Figure 3 where the data is normalized to the highest signal intensity observed in all of the experiments. The manufacturer-recommended pressure of $2.1 \times 10^{-5}$ torr was found to give the highest sensitivity for all gases in the range tested. At this pressure, the maximum sensitivity was obtained when Ar was used as the buffer gas. The sensitivity decreased somewhat at higher and lower buffer gas pressures. Helium and air exhibited similar trends to Ar over the range investigated, but produced overall lower sensitivities (33 and $8 \%$ lower, respectively). With no added buffer gas, the sensitivity was very poor $(94 \%$ lower than the maximum using Ar). Effects of the buffer gas on QITMS sensitivity have been considered recently with a MALDI source producing short pulses of ions [20]. Two possible consequences of the buffer gas can be postulated: (1) The buffer gas may affect the strong dependence of ion trapping on the rf phase during injection and (2) different buffer gases and pressures could lead to different levels of CID, therefore affecting the observed sensitivity. The first point, although not as significant a problem with continuous ion sources be- 


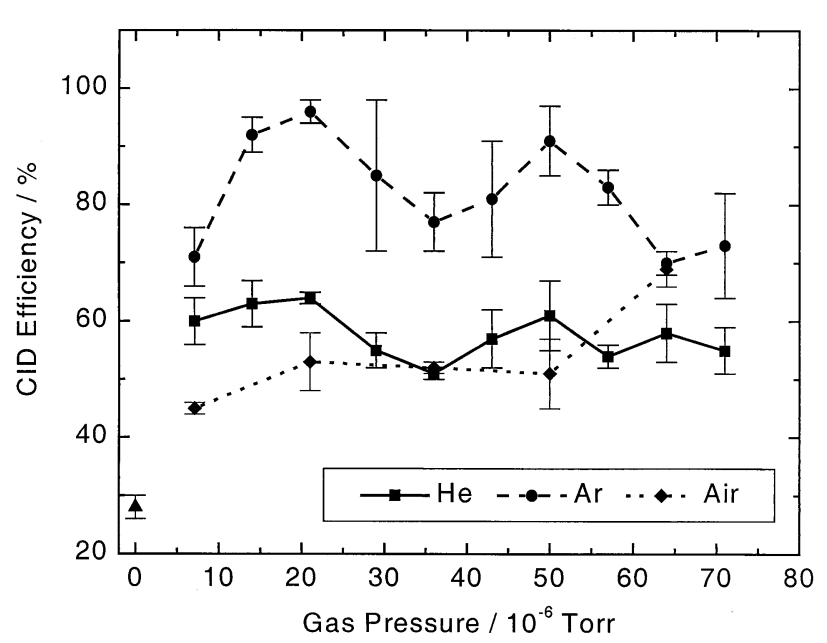

Figure 4. Collision-induced dissociation efficiency of the LeuEnk protonated molecule as a function of pressure for $\mathrm{He}, \mathrm{Ar}$, and Air.

cause the ions are injected over all rf phases, could be partially responsible for these results. However, the second point can be eliminated because upon injection argon produces only slightly more ion fragmentation than helium (see Figure 1). Therefore, additional unknown effects from the heavier buffer gas must be contributing to the sensitivity increase. Current work in our lab addressing the effect of the buffer gas during ion trapping in a QITMS is beginning to separate the many consequences [21].

\section{CID Performance}

After establishing that the Bruker Esquire would operate with static pressures of pure heavy gases (contrary to previous results with the Finnigan ITMS), the effects of heavy gas on CID were evaluated. The MS/MS (in this case CID) efficiency was calculated as follows [19]:

$$
\begin{aligned}
& \text { CID Efficiency }= \\
& \frac{\sum(\text { product ion signal after activation })}{\sum(\text { product ion signal before activation })}
\end{aligned}
$$

This efficiency was measured over the range of pressures of each gas, and the results are plotted in Figure 4. The manufacturer recommended pressure of $2.1 \times 10^{-5}$ torr was found to provide the maximum CID efficiency for He and Ar, with generally lower efficiencies being observed at pressures above and below this value. The CID efficiencies were relatively flat across the pressure range when air was used, with an increase observed at the highest pressures. The reason for this increase and the shape of the graph beyond this pressure was not investigated. The CID efficiency increased an average of $25 \%$ when Ar was used as compared to He. Air and He produced similar efficiencies over the pressure range examined. Despite the fact that air's molecular weight is closer to that of Ar than that of $\mathrm{He}$, a similarity between the air and He efficiencies exists, likely because air is comprised of polyatomic species whereas $\mathrm{He}$ and $\mathrm{Ar}$ are atomic species. A collision with a neutral polyatomic species can result in vibrational excitation of the neutral as well as the ion, whereas vibrational excitation is not possible for an atomic species. If the neutral can be vibrationally excited then less of the collision energy is likely to be converted into ion internal energy leading to ion dissociation. Again, as with ion trapping, CID was very inefficient when no buffer gas was added. This is expected because there is a relatively low number density of neutral gas molecules present for collisional energy transfer. Therefore, the parent ions are likely ejected before accumulating enough internal energy to dissociate.

In addition to the efficiency of generating product ions, the types of product ions formed from a CID experiment are important. The formation of certain product ion types is governed by the amount of internal energy deposited into the parent ion, which is modified by the presence of heavy gases within the QITMS [8, 13]. The ratio of $a_{4} / b_{4}$ ions formed from Leu-Enk was used to investigate the energy transfer process. The $\mathrm{a}_{4} / \mathrm{b}_{4}$ ion ratio has been shown previously to be indicative of the internal energy level of the dissociating protonated Leu-Enk [22]. The previous results showed that more internal energy is required to form the $a_{4}$ ion. Therefore, a higher $a_{4} / b_{4}$ ion intensity ratio indicates that the parent Leu-Enk ion possessed a larger amount of internal energy when it dissociated, and vice-versa. The $a_{4} / b_{4}$ ion ratio has been used previously in our lab with the QITMS to probe internal energy deposition [8]. Additional work in our lab established that the $\mathrm{a}_{4}$ ion from Leu-Enk is primarily formed from a consecutive dissociation as follows [23]: $[\mathrm{M}+\mathrm{H}]^{+} \rightarrow \mathrm{b}_{4} \rightarrow \mathrm{a}_{4}$.

The potential for a consecutive dissociation complicates the interpretation of the $a_{4} / b_{4}$ ion ratio as an indicator of internal energy deposition. Some recent publications have specifically challenged the use of the $\mathrm{a}_{4} / \mathrm{b}_{4}$ ion ratio as a measure of the internal energy of protonated Leu-Enk trapped within the QITMS [24, 25]. It was noted that if bath gas conditions, such as temperature, pressure and composition are not held constant, the consecutive dissociation of the $b_{4}$ ion to the $a_{4}$ ion can be affected through collisional cooling of the excited $b_{4}$ ion. Collisional cooling serves to decrease the ion internal and kinetic energy, therefore relaxing the ion to a lower energy state. $\mathrm{A} \mathrm{b}_{4}$ product ion that has internal energy in excess of the critical energy for dissociation to the $\mathrm{a}_{4}$ ion may not undergo dissociation if it is collisionally cooled (relaxed) at a rate faster than its dissociation rate. Rapid collisional cooling of the $b_{4}$ ion will divert the reaction from the reaction channel leading to the $\mathrm{a}_{4}$ ion, as was previously observed at low bath gas temperatures and low activation levels [25]. If the excited $b_{4}$ ion is not efficiently collisionally cooled the $a_{4}$ ion formation channel would be accessible, resulting in a large $a_{4} / b_{4}$ ratio. Therefore, the $a_{4} / b_{4}$ ion 


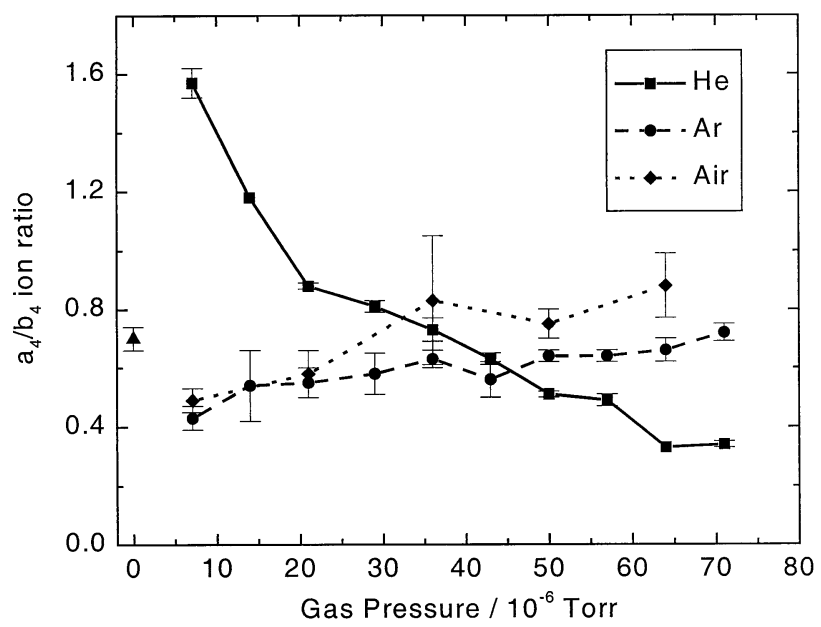

Figure 5. $a_{4} / b_{4}$ product ion ratio from the Leu-Enk protonated molecule as a function of pressure for $\mathrm{He}$, Ar, and Air.

ratio is affected by the internal energy of the dissociating parent ion, as well as the collisional cooling rate of the bath gas. In the work presented here the $a_{4} / b_{4}$ ion ratio was measured to gain information about the internal energy deposited into the parent ion and the collisional cooling rate of the $b_{4}$ product ions (and parent ions). However, it is impossible to deconvolute the specific contributions from the parent ion internal energy and the collisional cooling rates in these experiments (we are doing other experiments to separately measure collisional cooling rates [26]).

The $a_{4} / b_{4}$ ion intensity ratio results are shown in Figure 5. As with all of the MS/MS experiments, the amplitude of the resonance excitation voltage was increased until the product ion signal was maximized, yielding the maximum MS/MS efficiency. The most striking feature of Figure 5 is the large $a_{4} / b_{4}$ ion ratio observed with low pressures of $\mathrm{He}$, followed by a dramatic decrease in the ion ratio as the He pressure is increased. A subtle feature is the steady increase in the $a_{4} / b_{4}$ ion ratio as the pressure of Ar and air is increased.

The features of Figure 5 can be explained when the ion collisional cooling and dissociation rates are considered, as both affect the $a_{4} / b_{4}$ ion ratio. While on the microscopic level the processes are intertwined, on the macroscopic level for the sequential dissociation involved in the system under study, the key parameters are the dissociation rate of the parent ion $\left(\mathrm{k}_{\mathrm{pdis}}\right)$, the dissociation rate of the $b_{4}$ ion $\left(\mathrm{k}_{\mathrm{bdis}}\right)$ and the collisional cooling rate of the $b_{4}$ ion $\left(\mathrm{k}_{\mathrm{ccr}}\right)$. The overall relationship of these parameters is shown in Scheme 1 , where $b_{4}^{*+}$ is the excited $b_{4}$ ion that has enough internal energy to dissociate to the $a_{4}^{+}$ion. (Some fraction of the $b_{4}^{+}$ product ions will not have enough internal energy to subsequently dissociate and these are not considered further here.) Assuming only bimolecular collisions, the collisional cooling rate can be calculated as:

$$
\mathrm{k}_{\mathrm{ccr}}=\mathrm{k}_{\mathrm{cc}}[\text { buffer gas }]
$$

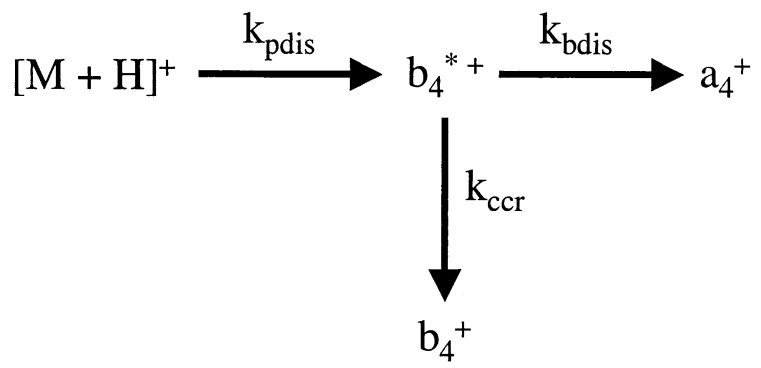

Scheme 1

where $\mathrm{k}_{\mathrm{cc}}$ is the collisional cooling rate constant $\left(\mathrm{cm}^{3} /\right.$ molecule $\left.\times \mathrm{s}\right)$ and [buffer gas] is the buffer gas number density (molecule $/ \mathrm{cm}^{3}$ ). The collisional cooling rate constant is different for each buffer gas because the size and type of neutral will affect the collision dynamics and therefore will affect how much ion internal energy is transferred to the neutral.

The above relationship can be used to explain why the $a_{4} / b_{4}$ ion ratio is so much greater with low pressures of He versus low pressures of heavy gases. The collisional cooling rate constant of He is much slower (due to its low mass and the fact that it is monoatomic) than that of either Ar or air. Therefore, $b_{4}^{*+}$ product ions dissociate faster than they are collisionally cooled by $\mathrm{He}$, leading to a larger amount of the $\mathrm{a}_{4}$ product ion being formed. As more He is added to the QITMS, $\mathrm{k}_{\mathrm{ccr}}$ increases and becomes more competitive with $\mathrm{k}_{\mathrm{bdis}}$ and the $a_{4} / b_{4}$ ion ratio decreases.

Collisional cooling, however, cannot explain why the $a_{4} / b_{4}$ ion ratio increases as the pressure of heavy gas increases. The ion ratio increase can be rationalized by remembering that the $a_{4} / b_{4}$ ion ratio is also a function of the dissociating parent ion internal energy. The critical energy of dissociation for the ion is the same, regardless of the buffer gas present. Once the critical energy is reached, there is still some finite time before the ion actually dissociates (the kinetic shift). During the time before dissociation the ion is still being resonantly excited and therefore it can undergo further activating collisions. There will be more collisions at the higher pressures of buffer gas (due to the shorter mean free path) serving to raise the internal energy of the activated parent ion to greater levels before it dissociates. With heavy gases, significantly more internal energy will be deposited than with $\mathrm{He}$, because of the higher $E_{\text {com }}$ value. When the highly activated parent ion dissociates, there will be more $b_{4}^{*+}$ product ions (relative to $b_{4}^{+}$product ions with insufficient internal energy to dissociate) and these $b_{4}^{*+}$ product ions will have additional internal energy relative to the same ions formed at lower Ar pressures. Thus, there are more ions that can dissociate to form $\mathrm{a}_{4}^{+}$product ions, and they require more collisional cooling to bring them below the critical energy of dissociation. The increase in the $a_{4} / b_{4}$ ion ratio observed at higher heavy gas pressures indicates that product ion internal energy (which should be proportional to the parent ion internal energy) increases 
faster than $\mathrm{k}_{\mathrm{ccr}}$. A similar increase is not observed with He because the parent ion internal energy is not raised as high as with heavier gases. For $\mathrm{He}$, in the pressure range tested, $\mathrm{k}_{\mathrm{ccr}}$ continues to increase faster than the internal energy of the $\mathrm{b}_{4}^{*+}$ ion.

A note should be made about the previous results obtained with the Finnigan ITMS concerning internal energy deposition during CID [6, 8]. The data from the earliest work [6] agrees very well with the findings presented here especially considering the relative pressures used. The previous researchers used lower pressures of pure He and pure Ar. Their data indicated a dramatic increase in the internal energy deposition at the lowest He pressures, agreeing with the conclusion above that $\mathrm{k}_{\mathrm{ccr}}$ is very slow at low pressures of He. All pressures of Ar tested produced little change in the parent ion internal energy, indicating that any increases in ion internal energy were matched by increases in $\mathrm{k}_{\mathrm{ccr}}$. The later work [8] found large $a_{4} / b_{4}$ ion ratios when CID was performed with $\mathrm{He}$ and a pulsed pressure of heavy gas present. The level of the ion ratio increase is particularly interesting relative to the small change observed here. The difference between the current and previous experiments appears to be that the heavy gas was pulsed into the QITMS, with the normal pressure of helium present. With two different gases in the QITMS, there are two contributions to $\mathrm{k}_{\mathrm{ccr}}$. Additionally, because the heavy gas is pulsed into the QITMS, its contribution to the collisional cooling rate will initially be large, but will then decrease as the heavy gas is pumped away. Apparently, the initial presence of heavy gas causes the internal energy of the parent ions to be raised well above the critical energy of dissociation. However, the heavy gas is pumped away when the $\mathrm{b}_{4}^{*+}$ product ions need to be collisionally cooled. With the heavy gas pumped away, all that is left to contribute to $\mathrm{k}_{\mathrm{ccr}}$ is the He. The $\mathrm{k}_{\mathrm{cc}}$ for He is much less than the $\mathrm{k}_{\mathrm{cc}}$ for a heavy gas, so $\mathrm{He}$ is not able to collisionally cool the product ions as efficiently. Therefore, the heavier gas is able to activate the ions, but does not compensate for the activation by additional collisional cooling. The pulsed introduction of the heavy gas in the previous work is what leads to the particularly high $a_{4} / b_{4}$ ion ratios.

The MS/MS process and the collision dynamics present also were investigated through the analysis of the resonance excitation voltage's relationship with buffer gas pressure. The resonance excitation voltage was optimized in each experiment such that the highest MS/MS efficiency was obtained. By considering the voltage required for each gas, at each pressure, it is possible to qualitatively assess the energetics involved in ion activation. Figure 6 shows the trend in resonance excitation voltage as a function of the pressure of each gas. Over the entire pressure range investigated, a higher resonant excitation voltage was required to maximize the MS/MS efficiency with Ar or air versus He. As noted above, the critical energy of dissociation

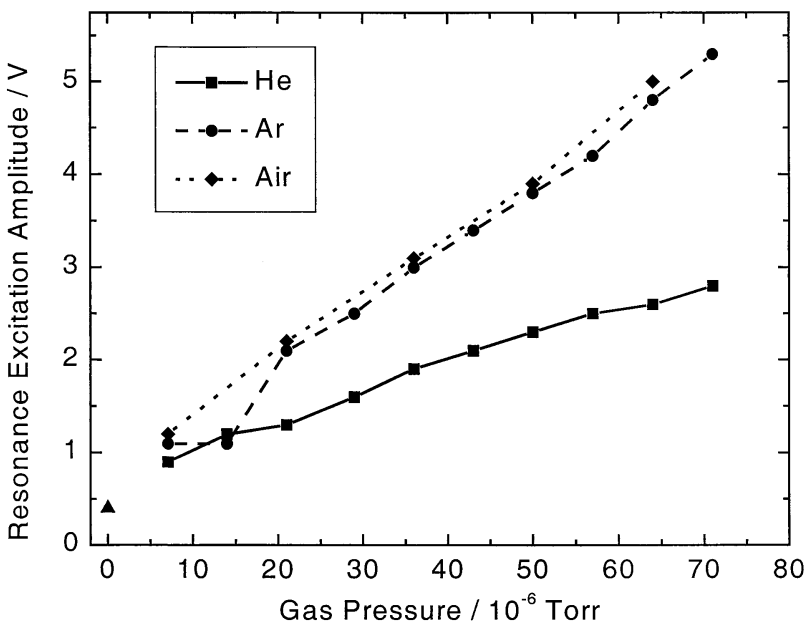

Figure 6. Resonance excitation amplitude required for maximum CID efficiency of the Leu-Enk protonated molecule as a function of pressure for $\mathrm{He}, \mathrm{Ar}$, and Air.

for the parent ion is the same regardless of the gas or pressure used. The resonance excitation voltage is essentially a measure of the "power" required to reach the critical energy for dissociation. Therefore, heavy gases would be expected to require less power as more energy can be transferred per collision $\left(\mathrm{E}_{\mathrm{com}}\right)$. During resonance excitation the goal is to increase the ion internal energy, however, the activated parent ion can still undergo collisional cooling. Although the parent ion will be immediately kinetically excited again after the cooling event, a collision that takes any energy away from the parent ion will slow the activation process. The difference in $\mathrm{k}_{\mathrm{cc}}$ for the parent ion is what produces the differences in the required resonance excitation voltage for $\mathrm{He}$ versus the heavy gases at a given pressure. Heavy gases require more voltage because $\mathrm{k}_{\mathrm{cc}}$ is greater.

In addition to differences in the required resonance excitation voltage with the gas type, the data shown in Figure 6 demonstrates that the required resonant excitation voltage increases for all gases as their pressure increases. As the gas pressure increases, $\mathrm{k}_{\mathrm{ccr}}$ will increase, regardless of gas type (as shown in eq 3). However, the collisional activation rate will also change with pressure, potentially canceling out the additional collisional cooling. It is, therefore, unknown if the $\mathrm{k}_{\mathrm{ccr}}$ change can explain the strong pressure dependence. The increase in the required resonance excitation voltage with buffer gas pressure is likely due to the decrease in the mean free path of the ion. For a given resonance excitation voltage, the smaller mean free path results in lower ion kinetic energies being reached before the next collision, because there is less time for the ion to absorb power. Therefore, to reach similar kinetic energies ( $E_{\text {lab }}$ in eq 1$)$ a larger excitation voltage is required as the pressure is increased, regardless of the gas used. 
Table 1. Leu-Enk $[\mathrm{M}+\mathrm{H}]^{+}$peak width (FWHM/) and intensitites observed with static pressures of Ar at two $\beta_{\text {eject }}$ values on the Finnigans ITMS

\begin{tabular}{lcclll}
\hline & \multicolumn{2}{c}{$\beta_{\text {eject }}=0.96$} & & \multicolumn{2}{c}{$\beta_{\text {eject }}=2 / 3$} \\
\cline { 2 - 3 } \cline { 6 - 6 } Pressure/torr & Width & Intensity & & Width & Intensity \\
\hline \hline $1 \times 10^{-3}$ & NS $^{a}$ & NS & & 6.12 & 142 \\
$5 \times 10^{-4}$ & 8.55 & 171 & & 2.34 & 217 \\
$1 \times 10^{-4}$ & 0.32 & 1125 & & 0.294 & 1187 \\
\hline
\end{tabular}

${ }^{a} \mathrm{NS}$ indicates no signal was observed.

\section{Instrument Dependencies}

All of the experiments discussed thus far were performed with the Bruker Esquire ion trap, which is considered a current generation QITMS. This instrument includes two features that may enable and/or enhance its operation with heavier buffer gases. First, the physical electrode geometry (both the spacing of the ring and endcaps and their shape) is specifically designed to enhance the contribution from higher order fields, particularly hexapolar fields [14, 15]. Second, the instrument detects ions by resonantly ejecting them at $\beta_{\mathrm{z}}=2 / 3$. At a $\beta_{\mathrm{z}}$ of $2 / 3$ ions are in resonance with the hexapolar field and are ejected more efficiently [27-29]. These two features should yield more efficient ejection under heavily damped conditions, such as when Ar is used as the buffer gas. The previous work with heavy gases was performed on Finnigan ITMS instruments, which are a previous generation of QITMS, which do not have the same electrode geometry and generally use a frequency for the resonance ejection voltage close to $\beta$ $=1[7-9,11,13]$.

To investigate the importance of the $\beta_{\mathrm{z}}$ value used for resonant ejection $\left(\beta_{\text {eject }}\right)$, additional experiments were conducted with a Finnigan ITMS. Operation of the ITMS with $1 \times 10^{-3}$ torr of Ar (the normal operating pressure when $\mathrm{He}$ is used) yielded no signal when operating at $\beta_{\text {eject }}$ near 1 (the normal resonant ejection point); this is in agreement with what has been observed previously $[7,8]$. Experiments were conducted with different Ar pressures and $\beta_{\text {eject }}$ values to determine what caused the loss in signal. Table 1 lists the peak widths and intensities observed. As indicated, signal was obtained at lower Ar pressures or at lower $\beta_{\text {eject }}$ values, but only after lowering the Ar pressure and operating at $\beta_{\text {eject }}=2 / 3$ did the peak widths and intensities approach those obtained with He. In general, operation of the ITMS over a range of static pressures of Ar resulted in signal intensity and resolution that are worse than those obtained with the Esquire. The adjustment of the $\beta_{\text {eject }}$ value alone did not cause the ITMS to perform as well as the Esquire. The only other difference between the ITMS and the Esquire is their electrode geometries, indicating that the higher order fields produced by the electrodes are also important in allowing acceptable operation with heavy gases.

Peak widths observed on the ITMS were recorded

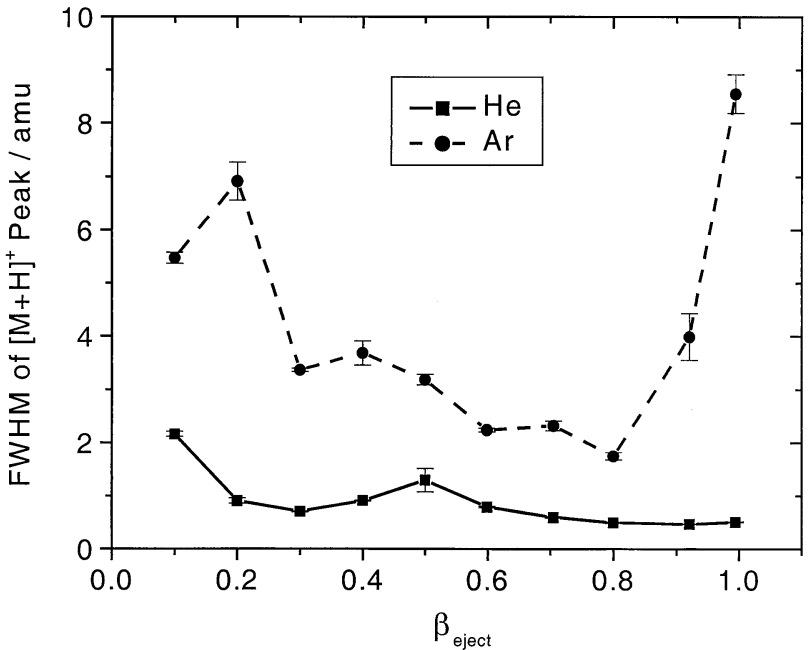

Figure 7. Leu-Enk protonated species peak width as a function of the $\beta_{\text {eject }}$ value. Data was obtained with $5 \times 10^{-4}$ torr of He or Ar on a Finnigan ITMS.

over a range of $\beta_{\text {eject }}$ values with both He and Ar buffer gases at a pressure of $5 \times 10^{-4}$ torr (one half the normal operating pressure). The results are shown in Figure 7. The peak widths are improved with Ar between $\beta_{\text {eject }}=$ 0.3 and 0.8. Peak widths increased for both gases as $\beta_{\text {eject }}$ decreased below 0.3 . This is expected because the separation of $m / z$ values along the $\mathrm{a}_{\mathrm{z}}=0$ axis of the stability diagram decreases as $\beta$ decreases. At $\beta_{\text {eject }}$ values above 0.8 , the peak widths increased rapidly when Ar was used, but continued to decrease with He. The optimum $\beta_{\text {eject }}$ value is, as expected, approximately 1 when $\mathrm{He}$ is used as the buffer gas. However, the optimum $\beta_{\text {eject }}$ value when operating with $\mathrm{Ar}$ is approximately 0.8 . Ejecting ions at $\beta$ values of $2 / 3$ or $1 / 2$ (the hexapolar and octopolar resonances, respectively) has been shown to produce very efficient ion ejection [27-29]. However, when these $\beta_{\text {eject }}$ values (and values closely surrounding them), were used with the ITMS, peak widths were not further reduced in the presence of $\mathrm{He}$ or $\mathrm{Ar}$ (data not shown). Possibly the faster ion ejection was mitigated by additional scattering or fragmentation of the quickly accelerated ions.

A final set of experiments to investigate the instrumental effects of heavy gases on QITMS operation was conducted with a Finnigan LCQ, another current generation QITMS. The geometry of the LCQ ion trap incorporates some higher order fields through increased trap stretching (accomplished by decreasing the ring electrode radius and maintaining the same endcap spacing) as compared to the ITMS [29, 30]. However, it is unknown if other fields are specifically enhanced with additional electrode geometry modifications. Operation of the LCQ under "normal" conditions with a static pressure of $\operatorname{Ar}\left(1 \times 10^{-3}\right.$ torr, $\left.\beta_{\text {eject }}=0.927\right)$ gave very broad peaks (much greater than the Esquire). Because the LCQ used for these experiments was modified so that the $\beta_{\text {eject }}$ value could be user-specified [16], 


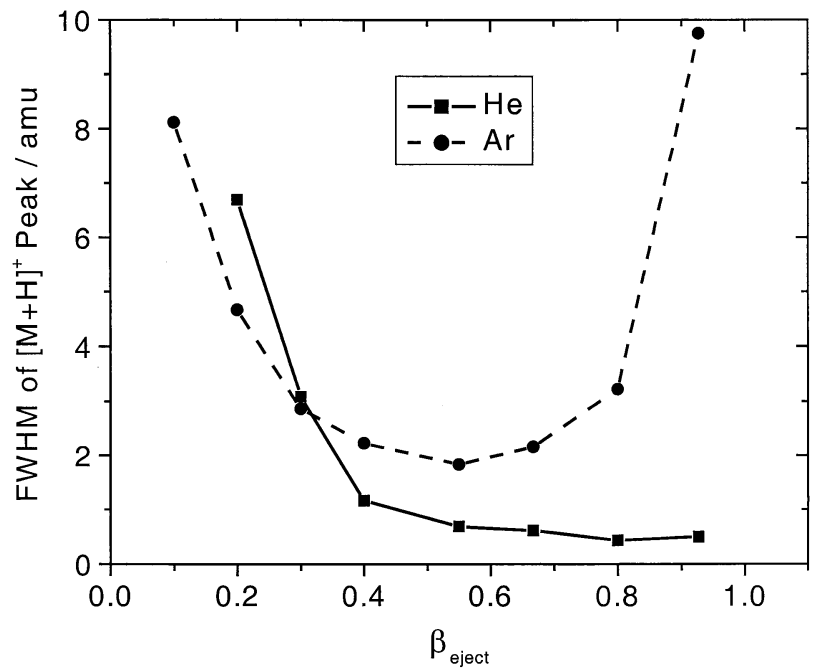

Figure 8. Leu-Enk protonated species peak width as a function of the $\beta_{\text {eject }}$ value. Data was obtained with $1 \times 10^{-3}$ torr of He or $\mathrm{Ar}$ on a Finnigan LCQ.

spectra were taken over a range of $\beta_{\text {eject }}$ values. Figure 8 shows the peak widths observed for both Ar and He. Similar trends are evident in Figures 7 and 8. Although the peak widths that were observed with the two instruments are similar, the ITMS experiments were conducted at one half the pressure that was used with the LCQ. Using the same buffer gas pressure with the ITMS would have produced very wide peaks and very little signal.

The above experiments demonstrate that the electrode geometry and the $\beta_{\text {eject }}$ value both play roles in optimization of the QITMS performance with heavy gases. The performance of the Finnigan ITMS compared to that of the Bruker Esquire shows that the presence of enhanced higher order fields improves QITMS performance with heavy gases, particularly with higher pressures of heavy gases. The performance of the LCQ, with its enhanced higher order fields, was superior to the performance of the ITMS with $1 \times 10^{-3}$ torr of Ar and $\beta_{\text {eject }}=0.96$. The resolution achieved with the LCQ was improved further when using Ar by lowering the $\beta_{\text {eject }}$ value used, which required modifications to the instrument. At lower $\beta_{\text {eject }}$ values the performance of the LCQ was very similar to the performance of the Bruker Esquire. The analytical mass scan rates used with these instruments, however, are not the same. A slower scan rate will yield higher resolution as the frequency resolution is greater [31]. The Esquire uses a scan rate that is more than two times faster than that used with the LCQ. Therefore, at similar scan rates it would be expected that the Esquire would have better resolution. The performance difference between the Esquire ion trap and the LCQ indicates that the higher order fields in the Esquire have a somewhat more positive effect on the resolution compared to the LCQ when operating with heavy gases. A possible explanation for the better performance of the Esquire is that the faster ion ejection reduces the effect of processes that degrade resolution when using heavy buffer gases. There is not enough data, however, to place a quantitative value on the different levels of performance as a function of higher order fields present.

\section{Conclusions}

Results presented here show that operation with only $\mathrm{Ar}$ as the buffer gas is not only possible but also can provide some advantages relative to He. The sensitivity and the CID efficiency are improved when Ar is used; however, the resolution is decreased. Thus, Ar appears to be beneficial during initial ion trapping and CID but not during the analytical scan. In addition to Ar, it was shown that operation of a QITMS with air as the buffer gas is possible. Air produced slightly improved sensitivity and CID efficiency compared to $\mathrm{He}$, and although the resolution was degraded, it was not as poor as with Ar.

MS/MS studies with Leu-Enk allowed the energetics of ion activation and collisional cooling by the buffer gas to be investigated. The different buffer gases used in this study were found to produce different collisional cooling rates. The heavier buffer gases cooled excited product ions faster than He did. As the pressure of the heavy buffer gas was increased, additional internal energy could be imparted into the parent ion bringing it further above its critical energy of dissociation. Once the highly activated parent ion dissociated, the product ion internal energy was also greater.

Finally, it was found that acceptable performance with heavy buffer gases was only possible when the QITMS was designed and operated such that higher order fields (above quadrupolar) were enhanced. The Bruker Esquire ion trap design and standard operation conditions are well suited to working with heavy gases. Under standard operating conditions, the Finnigan ITMS, and to a lesser extent the LCQ, are not well suited for use with heavy gases. However, both Finnigan instruments can be used effectively with heavy gases when operated with lower pressures and/or lower $\beta_{\text {eject }}$ values. Experiments with the ITMS and the LCQ demonstrated that the resolution depends on $\beta_{\text {eject }}$ and the functional form of the dependence is different when $\mathrm{Ar}$ is used, compared to He. With Ar as the buffer gas, the resolution improved drastically at $\beta_{\text {eject }}$ values between 0.4 and 0.8 . The expected resolution improvement as $\beta_{\text {eject }}$ approaches 1 was not observed, illustrating a key difference when operating the QITMS with Ar versus $\mathrm{He}$ as the buffer gas.

\section{Acknowledgments}

The authors thank Bruker Daltonics for providing the Esquire ion trap instrument used in this work. They also thank the Chemistry Division of the Naval Research Laboratory for use of their LCQ instrument. This work was supported by NIH grant GM49852. 


\section{References}

1. Neuhauser, W.; Hohenstatt, M.; Toschek, P. E.; Dehmelt, H. G. Visual Observation and Optical Cooling of Electrodynamically Contained Ions. Appl. Phys. 1978, 17, 123-129.

2. Fulford, J. E.; Hoa, D.-N.; Hughes, R. J.; March, R. E.; Bonner, R. F.; Wong, G. J. Radio-Frequency Mass Selective Excitation and Resonant Ejection of Ions in a Three-Dimensional Quadrupole Ion Trap. J. Vac. Sci. Technol. 1980, 17, 829-835.

3. Stafford, G. C. J.; Kelley, P. E.; Syka, J. E. P.; Reynolds, W. E.; Todd, J. F. J. Recent Improvements in and Analytical Applications of Advanced Ion Trap Technology. Int. J. Mass Spectrom. Ion Processes 1984, 60, 85-98.

4. McLuckey, S. A.; Glish, G. L.; VanBerkel, G. J. Multiple Stages of Mass Spectrometry in a Quadrupole Ion Trap Mass Spectrometer: Prerequisites. Int. J. Mass Spectrom. Ion Processes 1991, 106, 213-235.

5. Wells, J. M.; Plass, W. R.; Patterson, G. E.; Ouyang, Z.; Badman, E. R.; Cooks, R. G. Chemical Mass Shifts in Ion Trap Mass Spectrometry: Experiments and Simulations. Anal. Chem. 1999, 71, 3405-3415.

6. Gronowska, J.; Paradisi, C.; Traldi, P.; Vettori, U. A Study of Relevant Parameters in Collisional-activation of Ions in the Ion-trap Mass Spectrometer. Rapid Commun. Mass Spectrom. 1990, 4, 307-314.

7. McLuckey, S. A.; Glish, G. L.; Asano, K. G. Coupling of an Atmospheric-Sampling Ion Source with an Ion-Trap Mass Spectrometer. Anal. Chim. Acta 1989, 225, 25-35.

8. Vachet, R. W.; Glish, G. L. Effects of Heavy Gases on the Tandem Mass Spectra of Peptide Ions in the Quadrupole Ion Trap. J. Am. Soc. Mass Spectrom. 1996, 7, 1194-1202.

9. Morand, K. L.; Cox, K. A.; Cooks, R. G. Efficient Trapping and Collision-Induced Dissociation of High-Mass Cluster Ions Using Mixed Target Gases in the Quadrupole Ion Trap. Rapid Commun. Mass Spectrom. 1992, 6, 520-523.

10. Nourse, B. D.; Cox, K. A.; Morand, K. L.; Cooks, R. G. Collisional Activation of Pyrene and Anthracene in an IonTrap Mass Spectrometer. J. Am. Chem. Soc. 1992, 114, $2010-$ 2016.

11. Charles, M. J.; McLuckey, S. A.; Glish, G. L. Competition Between Resonance Ejection and Ion Dissociation During Resonant Excitation in a Quadrupole Ion Trap. J. Am. Soc. Mass Spectrom. 1994, 5, 1031-1041.

12. Qin, J.; Chait, B. T. Matrix-Assisted Laser Desorption Ion Trap Mass Spectrometry: Efficient Trapping and Ejection of Ions. Anal. Chem. 1996, 68, 2102-2107.

13. Doroshenko, V. M.; Cotter, R. J. Pulsed Gas Introduction for Increasing Peptide CID Efficiency in a MALDI/Quadrupole Ion Trap Mass Spectrometer. Anal. Chem. 1996, 68, 463-472.

14. Franzen, J.; Gabling, R.-H.; Heinen, G.; Weiss, G. Method for Mass-Spectroscopic Examination of a Gas Mixture and Mass Spectrometer Intended for Carrying Out This Method. U.S. Patent 5,028,777.

15. Franzen, J. Simulation Study of an Ion Cage with Superimposed Multipole Fields. Int. J. Mass Spectrom. Ion Processes 1991, 106, 63-78.
16. Vachet, R. W.; McElvany, S. W. Application of External Customized Waveforms to a Commercial Quadrupole Ion Trap. J. Am. Soc. Mass Spectrom. 1999, 10, 355-359.

17. McLuckey, S. A.; Berkel, G. J. V.; Glish, G. L. Reactions of Dimethylamine with Multiply Charged Ions of Cytochrome c. J. Am. Chem. Soc. 1990, 112, 5668-5670.

18. Barmess, J. E.; Georgiadis, R. M. Empirical Methods for Determination of Ionization Gauge Relative Sensitivities for Different Gases. Vacuum 1983, 33, 149-153.

19. Yost, R. A.; Enke, C. G.; McGilvery, D. C.; Smith, D.; Morrison, J. D. High Efficiency Collision-Induced Dissociation in an RF-Only Quadrupole. Int. J. Mass Spectrom. Ion Phys. 1979, 30 , 127-136.

20. Robb, D. B.; Blades, M. W. A Critical Investigation of the Effects of the Radio Frequency Potential on the Trapping of Externally Injected Ions in Ion Trap Mass Spectrometry. Rapid Commun. Mass Spectrom. 1999, 13, 1079-1087.

21. Danell, A. S.; Glish, G. L. unpublished.

22. Thibault, P.; Alexander, A. J.; Boyd, R. K.; Tomer, K. B. Delayed Dissociation Spectra of Survivor Ions from HighEnergy Collisional Activation. J. Am. Soc. Mass Spectrom. 1993, 4, 845-854.

23. Vachet, R. W.; Ray, K. L.; Glish, G. L. Origin of Product Ions in the MS/MS Spectra of Peptides in a Quadrupole Ion Trap. J. Am. Soc. Mass Spectrom. 1998, 9, 341-344.

24. Asano, K. G.; Butcher, D. J.; Goeringer, D. E.; McLuckey, S. A. Effective Ion Internal Temperatures Achieved via Boundary Activation in the Quadrupole Ion Trap: Protonated Leucine Enkephalin. J. Mass Spectrom. 1999, 34, 691-698.

25. Asano, K. G.; Goeringer, D. E.; Butcher, D. J.; McLuckey, S. A. Bath Gas Temperature and the Appearance of Ion Trap Tandem Mass Spectra of High-Mass Ions. Int. J. Mass. Spectrom. 1999, 190/191, 281-293.

26. Black, D. M; Payne, A. H.; Glish, G. L. Effects of Temperature and Pressure on the Collisional Cooling Rates in a Quadrupole Ion Trap. Proceedings of the 50th ASMS Conference on Mass Spectrometry and Allied Topics; Orlando, FL, June, 2002.

27. Franzen, J. The Non-Linear Ion Trap. Part 4. Mass Selective Instability Scan with Multipole Superposition. Int. J. Mass Spectrom. Ion Processes 1993, 125, 165-170.

28. Franzen, J.; Gabling, R.-H.; Schubert, M.; Wang, Y. In Practical Aspects of Ion Trap Mass Spectrometry. I. Fundamentals of Ion Trap Mass Spectrometry. March, R. E.; Todd, J. F. J., Eds.; CRC Press: Boca Raton, 1995; pp 49-167.

29. Wells, J. M.; Plass, W. R.; Cooks, R. G. Control of Chemical Mass Shifts in the Quadrupole Ion Trap Through Selection of Resonance Ejection Working Point and rf Scan Direction. Anal. Chem. 2000, 72, 2677-2683.

30. Schwartz, J. C.; Bier, M. E.; Taylor, D. M.; Zhou, J. Technological Aspects of a New LC/MS ${ }^{\mathrm{n}}$ Quadrupole Ion Trap. Proceedings of the 43rd ASMS Conference on Mass Spectrometry and Allied Topics; Atlanta, GA, 1995; p 1114.

31. Schwartz, J. C.; Syka, J. E. P.; Jardine, I. High Resolution on a Quadrupole Ion Trap Mass Spectrometer. J. Am. Soc. Mass Spectrom. 1991, 2, 198-204. 